\title{
JOINS AND INTERSECTIONS OF IDEALS OF COMPACT OPERATORS
}

\author{
ALLEN SCHWEINSBERG
}

\begin{abstract}
It is shown that certain well-known ideals of compact operators are the intersection of a decreasing, countable family of strictly larger ideals. Also, it is shown that if $T_{1}$ and $T_{2}$ are compact operators, neither of which lies in the principal ideal generated by the other, and if $g$ is an arbitrary countably generated ideal, then there exist ideals $g_{1}$ and $g_{2}$ such that $g \subseteq g_{1} \vee g_{2}$ and $T_{i} \notin g_{i}, i=1,2$.
\end{abstract}

1. Introduction. Let $\mathcal{H}$ be a separable, infinite-dimensional, complex Hilbert space, and let $\mathscr{E}(\mathcal{H})$ be the ring of bounded linear operators on $\mathcal{H}$. Each twosided ideal of $\mathscr{L}(\mathcal{H})$ contains $\mathscr{F}$, the ideal of finite rank operators, and is contained in $e$, the ideal of all compact operators.

In $\$ 2$ of this paper we investigate the question of which ideals of compact operators are the intersection of a decreasing countable family of strictly larger ideals. Among the ideals which will be shown to have this property are some familiar ones: all countably generated ideals, the $p$-Schatten ideals [5], and more generally the ideals ${ }_{0}(f)$ and $\delta(f)$ introduced in [1], and any norm ideal properly contained in $\mathcal{C}$ (thereby answering a question of Salinas $[4, \S 7]$ ).

A still open and possibly difficult problem of the same genre is whether $e$ is the join of two strictly smaller ideals. This question was posed in [1] and received further attention in [4]. It is known that the dual is false: $\mathscr{F}$ cannot be the intersection of two larger ideals. In fact, a slightly stronger statement can be made. Implicit in the proof of Theorem 4.3 in [3] is the result that if $T_{1}$ and $T_{2}$ are operators of infinite rank then there is an operator $S$ of infinite rank with the property that $S$ is contained in the intersection of any two ideals $q_{1}$ and $g_{2}$ such that $g_{i}$ contains $T_{i}, i=1,2$. The point to be stressed is that $S$ is independent of $g_{1}$ and $g_{2}$. We will show in $\S 3$ that the dual result is false.

In the sequel we will not deal directly with ideals of operators, but rather with ideal sets of sequences of real numbers. The work of Calkin [2] provides a lattice isomorphism from the complete distributive lattice of ideals in $\mathcal{E}(\mathcal{H})$ onto the complete distributive lattice of ideal sets. If $C$ is the collection of all nonnegative real sequences that tend to zero, then an ideal set $J$ is any subset of $C$ which satisfies:

Received by the editors July 16, 1975 and, in revised form, December 8, 1975.

AMS (MOS) subject classifications (1970). Primary 47B05, 46K05; Secondary 46A45.

(1) American Mathematical Society 1976 
(i) If $\left\{\lambda_{n}\right\} \in J$ and $\pi$ is any permutation of the natural numbers, then $\left\{\lambda_{\pi(n)}\right\} \in J$.

(ii) If $\left\{\lambda_{n}\right\} \in J$ and $\left\{\mu_{n}\right\} \in J$, then $\left\{\lambda_{n} \vee \mu_{n}\right\} \in J$.

(iii) If $\left\{\lambda_{n}\right\} \in J$ and $0 \leqslant \mu_{n} \leqslant \lambda_{n}$ for every $n$, then $\left\{\mu_{n}\right\} \in J$.

(iv) If $\left\{\lambda_{n}\right\} \in J$ and $\alpha>0$, then $\left\{\alpha \lambda_{n}\right\} \in J$.

Corresponding to an ideal $g$ of compact operators is the ideal set $J$ of sequences of eigenvalues (repeated according to multiplicity) of the positive operators in $\mathscr{f}$.

Observe that $C$ itself is the ideal set corresponding to the ideal of all compact operators, and the set $F$ of all finitely nonzero sequences in $C$ corresponds to the ideal of finite rank operators. As well-known examples of nontrivial ideal sets we have for $p>0$ the sets $C_{p}=\left\{\lambda \in C: \sum_{n} \lambda_{n}^{p}<\infty\right\}$. For $p \geqslant 1$ these correspond to the $p$-Schatten norm ideals $e_{p}$. In [1] Brown, Pearcy, and Salinas introduced much broader classes of ideal sets. They termed "admissible" any nondecreasing function $f$ defined on $[0, \infty)$ which satisfies $f(x)>0$ for $x>0$ and $f(0)=0=\lim _{x \rightarrow 0^{+}} f(x)$. Then the sets $S(f)=\left\{\lambda \in C: \Sigma_{n} f\left(\alpha \lambda_{n}\right)<\infty\right.$, for some $\left.\alpha>0\right\}$ and $D(f)=\{\lambda \in C$ : $\sum_{n} f\left(\alpha \lambda_{n}\right)<\infty$, for all $\left.\alpha>0\right\}$ are ideal sets with corresponding ideals $\delta(f)$ and $\mathscr{D}(f)$. Notice that $D(f)=S(f)=C_{p}$ if $f(x)=x^{p}$.

2. Intersections of ideals of compact operators. Most of the results cited in the introduction follow from the following very general theorem.

THEOREM 2.1. Let $f_{i}, i=1,2,3, \ldots$, be an arbitrary countable family of admissible functions and let $J$ be any ideal set such that $F \subseteq J \subseteq \vee_{i} S\left(f_{i}\right)$. Then $J$ is the intersection of a countable decreasing family of strictly larger ideal sets.

Proof. In [1] it was shown that $S(f) \vee S(g)=S(f \wedge g)$, so if we let $g_{i}=f_{1} \wedge f_{2} \wedge \cdots \wedge f_{i}$, we see that $\vee_{i} S\left(f_{i}\right)=\vee_{i} S\left(g_{i}\right)=\cup_{i} S\left(g_{i}\right)$ since $S\left(g_{i}\right)$ increases with $i$. Now let $\lambda$ be any monotone sequence in $J$ such that $\lambda_{n} \neq 0$ for all $n$. First we will define sequences $\lambda^{(k)}, k=1,2,3, \ldots$, such that $\lambda^{(k)} \wedge \lambda^{(m)}=\lambda$ if $k \neq m$, and $\lambda^{(k)} \notin \cup_{i} S\left(g_{i}\right)$ for any $k$. To this end choose a sufficiently large index $r_{1}$ so that $r_{1} g_{1}\left(\lambda_{1}\right) \geqslant 1$. Choose $r_{2}>r_{1}$, so that $r_{2} g_{2}\left(\lambda_{r_{1}} / 2\right) \geqslant 2$. Having chosen $r_{n}$ choose $r_{n+1}>r_{n}$ so that

$$
r_{n+1} g_{n+1}\left(\lambda_{r_{n}} /(n+1)\right) \geqslant(n+1) \text {. }
$$

Then let

$$
\begin{array}{ll}
\lambda_{n}^{(1)}=\lambda_{1} & \text { for } 1 \leqslant n \leqslant r_{1}, \\
\lambda_{n}^{(2)}=\lambda_{r_{1}} & \text { for } r_{1}+1 \leqslant n \leqslant r_{2}, \\
\lambda_{n}^{(1)}=\lambda_{r_{2}} & \text { for } r_{2}+1 \leqslant n \leqslant r_{3}, \\
\lambda_{n}^{(2)}=\lambda_{r_{3}} & \text { for } r_{3}+1 \leqslant n \leqslant r_{4}, \\
\lambda_{n}^{(3)}=\lambda_{r_{4}} & \text { for } r_{4}+1 \leqslant n \leqslant r_{5},
\end{array}
$$

defining in turn finite subsequences of $\lambda^{(1)}, \lambda^{(2)}, \lambda^{(1)}, \lambda^{(2)}, \lambda^{(3)}, \lambda^{(1)}, \lambda^{(2)}, \lambda^{(3)}$, 
$\lambda^{(4)}, \ldots$ For all other values of $k$ and $n$ we let $\lambda_{n}^{(k)}=\lambda_{n}$. Obviously $\lambda^{(k)} \wedge \lambda^{(m)}=\lambda$ if $k \neq m$. Also $\sum_{n} g_{i}\left(\alpha \lambda_{n}^{(k)}\right)=\infty$, because for each $k, i$, and $\alpha>0$ we can find an arbitrarily large integer $p$ for which $\sum_{n} g_{i}\left(\alpha \lambda_{n}^{(k)}\right)$ $\geqslant \sum_{n} g_{p}\left(\lambda_{n}^{(k)} / p\right) \geqslant p$. Thus, $\lambda^{(k)} \notin \cup_{j} S\left(g_{i}\right)$.

Now let $J_{k}=J \vee P\left(\lambda^{(k)}\right) \vee P\left(\lambda^{(k+1)}\right) \vee \cdots$, where $P(\mu)$ denotes the principal ideal set generated by a sequence $\mu$. So $J_{1} \supseteq J_{2} \supseteq \cdots \supseteq J$. Finally we show $\bigcap_{n} J_{n} \subseteq J$. Suppose $\mu \in \cap_{n} J_{n}$. Then $\mu \in J_{1}$, implying that for some indices $k_{i}$ such that $1 \leqslant k_{1}<k_{2}<\cdots<k_{n}, n<\infty, \mu \in J \vee P\left(\lambda^{\left(k_{1}\right)}\right)$ $\vee \cdots \vee P\left(\lambda^{\left(k_{n}\right)}\right)$. But $\mu \in J_{k_{n}+1}$ also, so $\mu \in J \vee P\left(\lambda^{\left(h_{1}\right)}\right) \vee \cdots \vee P\left(\lambda^{\left(h_{m}\right)}\right)$ for some indices $h_{i}$ such that $k_{n}+1 \leqslant h_{1}<h_{2}<\cdots<h_{m}, m<\infty$. We conclude that $\mu \in J$, since the $h$ 's and $k$ 's are distinct, and $P(\mu) \cap P(\nu)$ $=P(\mu \wedge \nu)$ for monotone sequences $\mu$ and $\nu$.

Corollary 2.2. Each of the following ideals is the intersection of a countable, decreasing family of strictly larger ideals:

(a) the ideals $\mathscr{D}(f)$ and $\delta(f)$ where $f$ is any admissible function,

(b) any countably generated ideal,

(c) eách p-Schatten ideal $e_{p}, p \geqslant 1$,

(d) $\cup_{p>0} e_{p}$.

THEOREM 2.3. Any norm ideal $g$ which is properly contained in $\mathcal{C}$ is the intersection of a countable, decreasing family of strictly larger ideals.

Proof. First we sketch needed facts about normed ideals (see $[4, \S 2]$ for details). If $T$ is any positive finite rank operator whose monotone sequence of eigenvalues is $\mu=\left(\mu_{1}, \mu_{2}, \ldots, \mu_{r}, 0,0, \ldots\right)$ define $\Phi(\mu)=\|T\|_{g}$. The function $\Phi$ is the symmetric norming function generated by the norm on $g$. It satisfies $\Phi(\mu) \leqslant \Phi(\nu)$ if $\mu_{n} \leqslant \nu_{n}$ for all $n$. Its domain can be extended to include all monotone sequences in $C$ by setting $\Phi(\lambda)=\lim _{r \rightarrow \infty} \Phi\left({ }_{r} \lambda\right)$ where ${ }_{r} \lambda=\left(\lambda_{1}\right.$, $\left.\lambda_{2}, \ldots, \lambda_{r}, 0,0, \ldots\right)$. The set $\{\lambda \mid \Phi(\lambda)<\infty\}$ generates an ideal set, and the corresponding ideal $\mathfrak{\pi}_{\Phi}$ is the maximal norm ideal generated by $\Phi$. Since $g$ is properly contained in $\mathcal{C}, \Re_{\Phi}$ will be also. If $u$ is the sequence $(1,1,1, \ldots)$ then $\lim _{r \rightarrow \infty} \Phi\left({ }_{r} u\right)=\infty$ since the identity operator is not compact.

Now let $\lambda$ be a monotone sequence corresponding to an infinite rank

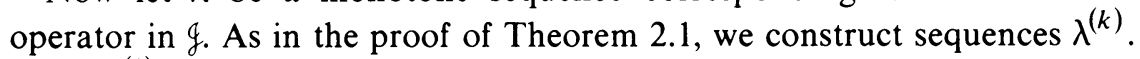

Let $\lambda_{n}^{(1)}=\lambda_{1}$ for $1 \leqslant n \leqslant r_{1}$ where $r_{1}$ is chosen so that $\lambda_{1} \Phi\left(r_{1} u\right) \geqslant 1$.

Let $\lambda_{n}^{(2)}=\lambda_{r_{1}}$ for $r_{1}+1 \leqslant n \leqslant r_{2}$ where $r_{2}>r_{1}$ is chosen so that $\lambda_{r_{1}} \Phi\left({ }_{r_{2}} u\right)$ $\geqslant 2$.

Continue defining finite subsequences of $\lambda^{(1)}, \lambda^{(2)}, \lambda^{(1)}, \lambda^{(2)}, \lambda^{(3)}, \ldots$ as in the proof of Theorem 2.1, and let $\lambda_{n}^{(k)}=\lambda_{n}$ for all other values of $k$ and $n$. Then $\lambda^{(k)} \wedge \lambda^{(m)}=\lambda$ if $k \neq m$ and $\Phi\left(\lambda^{(k)}\right)=\infty$ for each $k$ since $\Phi\left(\lambda^{(1)}\right) \geqslant 1$, $\Phi\left(\lambda^{(2)}\right) \geqslant 2, \Phi\left(\lambda^{(1)}\right) \geqslant 3, \Phi\left(\lambda^{(2)}\right) \geqslant 4, \Phi\left(\lambda^{(3)}\right) \geqslant 5$, etc. Thus $\lambda^{(k)} \notin \mathfrak{N}_{\Phi}$ and the remainder of the proof is identical to that of Theorem 2.1.

3. Joins of ideals of compact operators. Here we prove the following theorem.

THEOREM 3.1. Let $T_{1}$ and $T_{2}$ be compact operators, neither of which belongs to 
the principal ideal generated by the other. If $S_{k}, k=1,2,3, \ldots$, is an arbitrary countable collection of compact operators, then there exist ideals $g_{1}$ and $g_{2}$ such that $T_{i} \notin g_{i}, i=1,2$, but $S_{k} \in g_{1} \vee g_{2}$ for each $k$.

Proof. If $\mu \in C$ and $\eta>0$, let $\mu(\eta)$ be the number of indices $n$ for which $\mu_{n}>\eta$. Then it is known that $\mu \notin P(\lambda)$ if and only if $\lim \sup _{\eta \rightarrow 0} \mu(\eta) /(\alpha \lambda)(\eta)$ $=\infty$ for each $\alpha>0$ [6, Theorem 2.2].

Let $\lambda$ and $\mu$ belong to $C$ with $\lambda \notin P(\mu)$ and $\mu \notin P(\lambda)$. Let $\nu$ be any monotone sequence in $C$. We will exhibit sequences $\nu^{\prime}$ and $\nu^{\prime \prime}$ such that $\mu \notin P\left(\lambda \vee \nu^{\prime}\right), \lambda \notin P\left(\mu \vee \nu^{\prime \prime}\right)$, and $\nu=\nu^{\prime} \vee \nu^{\prime \prime}$, showing that the theorem is true in the special case of a single compact operator $S$.

Select an $\eta_{1}$ for which $\lambda\left(\eta_{1}\right) / \mu\left(\eta_{1}\right)>1$ and an index $k_{1}$ so that $\nu_{k_{1}} \leqslant \eta_{1}$.

Then select an $\eta_{2}$ for which $\mu\left(\eta_{2}\right) /\left((2 \lambda)\left(\eta_{2}\right)+k_{1}\right)>2$ and an index $k_{2}$ so that $k_{2}>k_{1}$ and $2 v_{k_{2}} \leqslant \eta_{2}$.

Select an $\eta_{3}$ for which $\lambda\left(\eta_{3}\right) /\left((3 \mu)\left(\eta_{3}\right)+k_{2}\right)>3$ and an index $k_{3}$ so that $k_{3}>k_{2}$ and $3 v_{k_{3}} \leqslant \eta_{3}$, and continue in this fashion selecting $\eta$ 's and $k$ 's. Finally let $k_{0}=0$ and define $\nu^{\prime}$ and $\nu^{\prime \prime}$ as follows:

for $k_{2 m}+1 \leqslant n \leqslant k_{2 m+1}$, let $\nu_{n}^{\prime}=\nu_{n}$ and $\nu_{n}^{\prime \prime}=0$, and

for $k_{2 m+1}+1 \leqslant n \leqslant k_{2 m+2}$, let $\nu_{n}^{\prime}=0$ and $\nu_{n}^{\prime \prime}=\nu_{n}, m=0,1,2, \ldots$

Consequently $\lambda\left(\eta_{1}\right) /\left(\mu \vee \nu^{\prime \prime}\right)\left(\eta_{1}\right)=\lambda\left(\eta_{1}\right) / \mu\left(\eta_{1}\right)>1$ since $\nu^{\prime \prime}\left(\eta_{1}\right)=0$. Also, $\mu\left(\eta_{2}\right) /\left(2 \lambda \vee 2 \nu^{\prime}\right)\left(\eta_{2}\right)>2$ since $\left(2 \nu^{\prime}\right)\left(\eta_{2}\right) \leqslant k_{1}$. And

$$
\lambda\left(\eta_{3}\right) /\left(3 \mu \vee 3 \nu^{\prime \prime}\right)\left(\eta_{3}\right)>3
$$

since $\left(3 \nu^{\prime \prime}\right)\left(\eta_{3}\right) \leqslant k_{2}$, etc. Thus,

$$
\limsup _{\eta \rightarrow 0} \lambda(\eta) /\left(\alpha \mu \vee \alpha \nu^{\prime \prime}\right)(\eta)=\limsup _{\eta \rightarrow 0} \mu(\eta) /\left(\alpha \lambda \vee \alpha \nu^{\prime}\right)(\eta)=\infty
$$

for each $\alpha>0$, implying that $\lambda \notin P\left(\mu \vee \nu^{\prime \prime}\right)$ and $\mu \notin P\left(\lambda \vee \nu^{\prime}\right)$. Obviously $\nu=\nu^{\prime} \vee \nu^{\prime \prime}$, so the proof is complete for this special case. If, in general, we have countably many monotone sequences $\nu_{(k)}, k=1,2,3, \ldots$, we can continue the above process inductively and obtain for each $k$ the principal ideal sets

$$
P_{k}^{\prime}=P\left(\lambda \vee \nu_{(1)}^{\prime} \vee \cdots \vee \nu_{(k)}^{\prime}\right)
$$

and

$$
P_{k}^{\prime \prime}=P\left(\mu \vee \nu_{(1)}^{\prime \prime} \vee \cdots \vee \nu_{(k)}^{\prime \prime}\right)
$$

with $\nu_{(k)}=\nu_{(k)}^{\prime} \vee \nu_{(k)}^{\prime \prime}, \mu \notin P_{k}^{\prime}$, and $\lambda \notin P_{k}^{\prime \prime}$. Let $P^{\prime}=\cup_{k} P_{k}^{\prime}$ and $P^{\prime \prime}$ $=\cup_{k} P_{k}^{\prime \prime}$. Then $\nu_{(k)} \in P^{\prime} \vee P^{\prime \prime}$ for each $k$, while $\mu \notin P^{\prime}$ and $\lambda \notin P^{\prime \prime}$, completing the proof.

ADDED IN PROOF. The author has recently learned that Andreas Blass and Gary Weiss have shown, assuming the Continuum Hypothesis, that $C$ is the join of two proper ideals. If the compact operators are well-ordered so that 
each has at most countably many predecessors, then that result can also be obtained by using Theorem 3.1.

\section{REFERENCES}

1. A. Brown, C. Pearcy and N. Salinas, Ideals of compact operators on Hilbert space, Michigan Math. J. 18 (1971), 373-384. MR 45 \#909.

2. J. W. Calkin, Two sided ideals and congruences in the ring of bounded operators in Hilbert space, Ann. of Math. (2) 42 (1941), 839-873. MR 3, 208.

3. N. Salinas, Ideals sets and ideals of compact operators on Hilbert space, Indiana Univ. Math. J. 22 (1972/73), 505-521. MR 47 \#642.

4. - Symmetric norm ideals and relative conjugate ideals, Trans. Amer. Math. Soc. 188 (1974), 213-240. MR 49 \#1146.

5. R. Schatten, Norm ideals of completely continuous operators, Ergebnisse Math. Grenzgebiete, Heft 27, Springer-Verlag, Berlin, 1960. MR 22 \#9878.

6. A. Schweinsberg, Principal ideals of compact operators, Indiana Univ. Math. J. 25 (1976), 229-233.

Department of Mathematics, Bucknell University, Lewisburg, Pennsylvania 17837 